\title{
Head-to-head domain-wall phase diagram in mesoscopic ring magnets
}

\author{
M. Kläui \\ Fachbereich Physik, Universität Konstanz, Universitätsstr. 10, D-78457 Konstanz, Germany \\ C. A. F. Vaz and J. A. C. Bland ${ }^{\mathrm{a})}$ \\ Cavendish Laboratory, University of Cambridge, Madingley Road, Cambridge, CB3 OHE, \\ United Kingdom \\ L. J. Heyderman \\ Laboratory for Micro- and Nanotechnology, Paul Scherrer Institut, CH-5232 Villigen PSI, Switzerland \\ F. Nolting \\ Swiss Light Source, Paul Scherrer Institut, CH-5232 Villigen PSI, Switzerland
}

A. Pavlovska and E. Bauer

Department of Physics and Astronomy, Arizona State University, Tempe, Arizona 85287-1404

S. Cherifi, S. Heun, ${ }^{\text {b) }}$ and A. Locatelli

Sincrotrone Trieste, 34012 Basovizza, Trieste, Italy

(Received 3 May 2004; accepted 7 October 2004)

\begin{abstract}
The nanoscale spin structure of head-to-head domain walls in mesoscopic ferromagnetic rings has been studied by high-resolution nonintrusive photoemission electron microscopy as a function of both ring width (100-730 nm) and film thickness (2-38 nm). Depending on the geometry, two types of head-to-head domain walls are found (vortex and transverse walls). The experimental phase diagram, which identifies the transition between the wall types, is compared to analytical calculations of the energy and micromagnetic simulations, which are found to agree well with the experimental results. (C) 2004 American Institute of Physics. [DOI: 10.1063/1.1829800]
\end{abstract}

Domain walls are one of the fundamental features of magnetic systems and although a reasonably good understanding of the different types of domain walls occurring in the bulk and continuous films (Bloch, Néel, etc.) ${ }^{1}$ has been developed, domain walls that are geometrically confined in magnetic nanostructures have only recently become the focus of intense interest. ${ }^{2-5}$ Due to geometrical constraints, different types of walls (such as $180^{\circ}$ head-to-head walls) occur, and the spin structure of these walls can be dominated by the geometry rather than by the material properties as in the case of the bulk. ${ }^{3,5-9}$ This means that the domain-wall properties can be easily tailored by changing the geometrical parameters, and also by, e.g., including notches. ${ }^{8,10}$ From a fundamental physical point of view, the domain-wall types result from a minimization of the energetics governing the magnetization configurations in small magnetic structures (exchange, magnetostatic, and anisotropy). Thus, studying the geometry dependence of the domain-wall types, provides a deeper insight into the interplay between the different energy terms. Micromagnetic calculations allow these energy contributions to be quantified and comparison with the experiment leads to a better understanding of the energy landscape involved (the formation of domain walls sometimes includes nucleation or pinning processes, which leads to multiple local energy minima). Theoretically, two types of head-to-head domain walls (transverse walls and vortex walls) were predicted and systematically studied by McMichael and Donahue ${ }^{11}$ but so far no systematic experi-

\footnotetext{
a) Author to whom correspondence should be addressed; electronic mail: jacb1@phy.cam.ac.uk

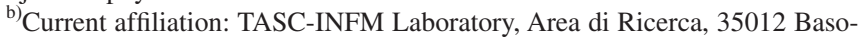
vizza (TS), Italy.
}

mental results have been made available for comparison and to check the validity of the calculations.

From an applications point of view, spintronic devices with switching via current-induced (rather than field induced) head-to-head domain-wall propagation are currently being heavily investigated, since they entail simple fabrication combined with possible fast and reliable switching. ${ }^{12,13}$ The performance of these devices depends crucially on the type and spin structure of the domain walls and thus a thorough understanding of the domain-wall evolution with changing geometrical parameters is necessary to develop and optimize these devices.

To study the transition between the two types of headto-head domain walls, ring structures are a useful geometry since they allow the creation and selective positioning of domain walls by saturating a ring along a certain direction. After relaxing the field to remanence, most rings will exhibit a head-to-head and a tail-to-tail wall positioned along the direction the field was applied. ${ }^{2,14-16}$ This magnetization configuration has been termed the "onion" state and is found to be a stable state in rings in addition to the flux-closure vortex state. $^{2,7,14-16}$ This straightforward manipulation of domain walls in rings is in sharp contrast to the case of straight geometries, where it is difficult to control the position of a domain wall using a uniform external field. Our method of creating the domain walls yields very reproducible domainwall types, which we confirmed here by establishing that the domain-wall types are always the same for a certain ring geometry even if the field is applied along different directions. Other methods, such as injecting domain walls from pads into straight wires with constrictions to pin the domain walls, have shown to sometimes yield different domain-wall spin structures for the same wire geometry, depending on the 

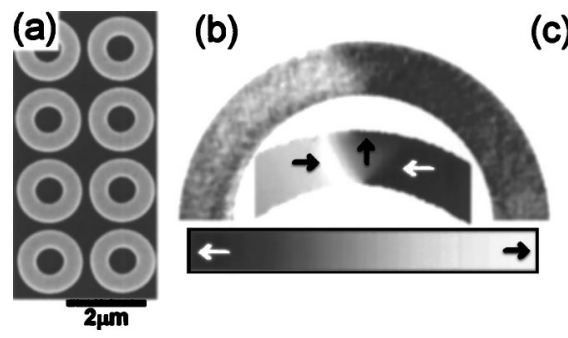

(c)

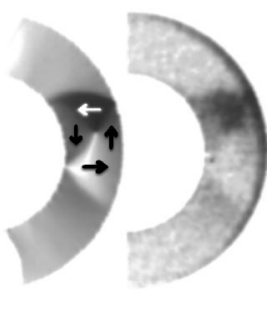

FIG. 1. (a) Scanning electron microscopy image of part of an array of polycrystalline Co rings (outer diameter $D=1.65 \mu \mathrm{m}$, width $W=530 \mathrm{~nm}$ and thickness $t=34 \mathrm{~nm}$ ). High-resolution PEEM images and corresponding micromagnetic simulations of parts of Co rings with $W=260 \mathrm{~nm}, t=10 \mathrm{~nm}$ exhibiting transverse walls (b) and $W=350 \mathrm{~nm}, t=34 \mathrm{~nm}$ exhibiting vortex walls (c). The greyscale bar and the arrows indicate the magnetization directions.

exact geometry of the constriction and also on the shape and connection of the wire and pad. ${ }^{17}$

In this letter, we report the results of a combined experimental and theoretical study of the transition between the vortex and transverse wall types as a function of the geometrical parameters. We show that the transition can be explained by the interplay of the energy terms involved, and comparison with micromagnetic simulations and analytical calculations allows us to evaluate the energy landscape.

Arrays of polycrystalline Co rings with $1.65 \mu \mathrm{m}$ outer diameter, widths $W$ of $110 \mathrm{~nm}-730 \mathrm{~nm}$, and thicknesses $t$ between $2 \mathrm{~nm}$ and $38 \mathrm{~nm}$ were fabricated by electron-beam lithography with lift off as described in Refs. 18 and 19. An edge-to-edge spacing of $3.5 \mu \mathrm{m}$ was used in order to prevent dipolar interactions. A scanning electron microscopy image of rings fabricated with this technique (albeit with a narrower spacing, which allows us to show a number of rings with high resolution) is presented in Fig. 1(a). To determine the domain-wall type, the ring arrays were directly imaged with nonintrusive $\mathrm{x}$-ray magnetic circular dichroism photoemission electron microscopy (X-PEEM). In X-PEEM, the yield of secondary electrons created by excitation with circularly polarized light depends on the dot product $\mathbf{P} \cdot \mathbf{M}$ of the helicity $\mathbf{P}$ and the magnetization direction $\mathbf{M}$. The secondary electrons are used for imaging by a photoemission electron microscope (PEEM) and the yield difference is visible as magnetic contrast in the images. ${ }^{20}$ To understand the contribution of the different energy terms, micromagnetic simulations were computed using the $\mathrm{OOMMF}^{21}$ package. The parameters used are: $M_{s}=1424 \times 10^{3} \mathrm{~A} / \mathrm{m}, \quad A=3.3$ $\times 10^{-11} \mathrm{~J} / \mathrm{m}$, a damping constant of 0.01 , and a cell size of 2-5 nm. In Fig. 1(b), a PEEM image and the corresponding micromagnetic simulation of a narrow and thin ring exhibiting transverse walls are shown. The triangular structure characteristic of a transverse wall is clearly visible and comparison with the micromagnetic simulation yields good agreement. Compared with transverse walls in straight wires, where the triangular structure is symmetry breaking and thus prone to irregularities, ${ }^{11}$ here, the symmetry-breaking curvature removes this extra degree of freedom leading to a very reproducible spin structure. As seen in Fig. 1(c), a wide and thick ring exhibits vortex walls. The vortex structure leads to an unambiguous circular black-white contrast that allows us to easily identify this type of wall. Again, micromagnetic simulations reproduce the spin structure very well.

The geometry dependence of the domain-wall type is determined by the interplay between the different contribut-
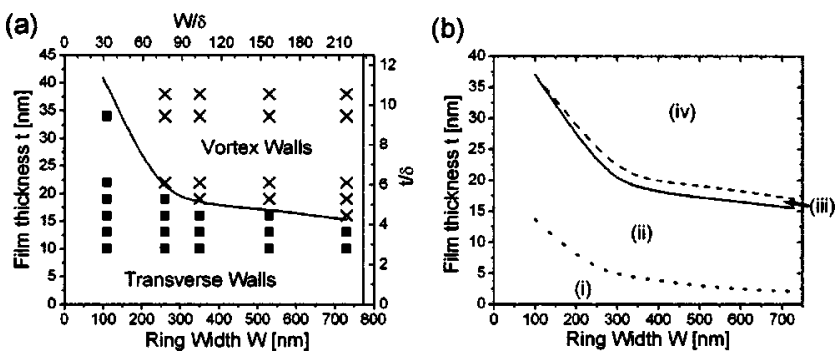

FIG. 2. (a) Experimental phase diagram for polycrystalline Co rings with outer diameter $D=1.65 \mu \mathrm{m}$ (squares: Transverse walls, crosses: Vortex walls, black solid curve: Phase boundary between the two wall types as a guide for the eye). The vertical line at $825 \mathrm{~nm}$ indicates the geometrical boundary between rings and disks and the widths and thicknesses are also given universally scaled to $\mathrm{W} / \delta$ and $t / \delta(\delta=3.6 \mathrm{~nm})$. (b) The experimental phase boundary (black solid curve) is shown together with the theoretical calculation of the boundary where the energies of the two wall types are equal (black dotted line as calculated from Ref. 11) and the phase boundary obtained from micromagnetic simulations of the wall types found after quasi-static relaxation of an applied field (black dashed line). The differences can be understood by looking at the four regions separated by the three lines: (i) The transverse wall is the lowest-energy configuration; (ii) the vortex wall is the lowest-energy configuration but the transverse wall constitutes a local energy minimum; (iii) the vortex wall is the lowestenergy configuration, the transverse wall constitutes a local energy minimum, and the energy barrier in between is small enough so it can be overcome by thermal fluctuations; and (iv) the vortex wall is the lowest-energy configuration and the transverse wall does not constitute an energy minimum.

ing energy terms: Exchange, magnetostatic (stray field), and magenetocrystalline anisotropy (which is averaged out to zero in these polycrystalline rings). Comparing the two domain-wall types qualitatively, it is obvious that the transverse wall has a high stray-field energy, while the vortex wall has less stray field but it has a vortex core, which is highly energetic due to the exchange interaction between strongly twisted spins. Since the exchange energy is linear in the film thickness, while the stray-field energy increases quadratically with film thickness, thin films favor transverse walls, while in thick-film structures vortex walls are expected. ${ }^{11}$ Moreover, the stray field energy of the transverse wall increases more strongly with increasing width than the exchange energy of the vortex wall; so that narrow rings exhibit transverse walls, while in wide rings vortex walls are expected. ${ }^{11}$

Since our micromagnetic simulations suggest that the curvature of the ring (effectively the outer diameter) has little influence on the wall type for the geometries investigated here (which allows us to compare our experimental results to calculations for, e.g., straight geometries), we have fixed the outer diameter to $1.65 \mu \mathrm{m}$ and varied the other two geometrical parameters, which are film thickness and ring width. The analysis of the spin structure of ring arrays with 40 different combinations of thickness and width leads to the quantitative phase diagram of the transition between vortex and transverse walls shown in Fig. 2(a), where indeed transverse walls are observed for thin and narrow rings and vortex walls for wide and thick rings. In Fig. 2(b), the experimental phase boundary (black solid line) is compared with a theoretical calculation (black dotted line). In the theoretical calculation according to Ref. 11, the energy for a vortex and a transverse wall is computed for a given geometry and the phase boundary where both energies become equal is found to be of the form $t \times W=C \times \delta^{2}$ with $\delta$ as the exchange length and $C$ as a universal constant. With the exchange 
length calculated as $\delta=\sqrt{A / \mu_{0} \times M_{s}^{2}}$ for cobalt using the constants mentioned above and $C$ from Ref. 11, it can be seen that the theoretical phase boundary has qualitatively the same shape as the experimental one, but the transition from transverse to vortex walls occurs in the experiment for much higher thicknesses and larger widths than theoretically predicted. To understand the discrepancy, we have to take into account the fact that in the theoretical calculation the total energies of the two wall types were computed and compared for a certain geometry, while in the experiment the wall formed after relaxing the field from saturation is observed (which might not necessarily constitute the lowest-energy configuration). When the field is relaxed, the first accessible energy minimum is the transverse wall, which is formed reversibly from saturation. ${ }^{8}$ The formation of the vortex wall requires the nucleation of a vortex core and it is well known that vortex nucleation occurs hysteretically, ${ }^{8,22}$ i.e., involving an energy landscape with different local energy minima separated by energy barriers. Since in most rings the global energy minimum at remanence is not the onion state but the flux-closure vortex state, we cannot obtain the onion state with the head-to-head walls with the lowest energy by demagnetizing the sample. Thus, the experimentally relevant wall type in the onion state is obtained after relaxing the field as done here. From this, it is clear that in order to reproduce the experimental results we have to model the experimental procedure by computing the wall type found in a ring after relaxing an applied field, rather than by looking at the total energy of the two domain-wall types for a given geometry. To simulate the quasi-static relaxation of the field to zero, we have reduced the field in steps $(20$ steps from $10 \mathrm{kOe}$ to 1 kOe, 20 steps from $1 \mathrm{kOe}$ to $100 \mathrm{Oe}$, and 50 steps from 100 Oe to $0 \mathrm{Oe}$ ) and waited after each step until the maximum torque was below $10^{-4}$ (if the field is relaxed from $10 \mathrm{kOe}$ to zero in a single field step, dynamic effects may lead to wrong results). For some geometries, we checked that neither reducing the cell size, nor using half rings with just one wall, changes the resulting wall type. The phase boundary computed this way is shown as the dashed black line in Fig. 2(b) and it reproduces the experimental results very closely. It is shifted to slightly larger thicknesses and widths compared to the experimentally found boundary (full line). If significant, a possible reason for the shift might lie in the fact that the simulations were carried out at $0 \mathrm{~K}$, while the experiments on the other hand were carried out at room temperature, where for certain geometries thermal excitations can overcome small energy barriers and thus switch the transverse wall (which constitutes a local energy minimum) to the vortex wall (which is the lower-energy minimum) for sufficiently small energy barriers. ${ }^{23}$ Other effects that might contribute to this shift are, e.g., defects such as edge roughness, which are not included in the model, though we would expect that defects rather shift the experimental boundary up (contrary to what we observe). To clarify this, further temperature dependent measurements and simulations are needed.

In conclusion, we have studied the phase transition from vortex to transverse walls experimentally and theoretically for micrometer-sized polycrystalline Co rings. Comparison of the experimental phase diagram with analytical calculations of the total energy of the different domain-wall types for a certain geometry, shows qualitatively similar phase boundaries. The absolute values for the critical width and thickness for the transition are much higher in the experiment since the formation of vortex walls entails a vortex core nucleation and hence local energy minima for both wall types separated by an energy barrier. Micromagnetic simulations of the experimental procedure (quasi-static field reduction) reproduce the experimental values very closely and the small differences could be due to thermal exciations indicating the energy barrier heights between the different wall types.

This work was supported by the CMI Magnetoelectronic Devices project, the DAAD [for one of the authors (M.K.)], and the "Deutsche Forschungsgemeinschaft" (SFB 513). One of the authors (E.B.) acknowledges support by NSF (Grant No. 9818296) and ONR (Grant No. 000140210922). Part of this work (data for Fig. 2) was carried out at the Swiss Light Source, Paul Scherrer Institut (Villigen, Switzerland), and the other part (Fig. 1) at the Nanospectroscopy Beamline at Elettra (Trieste, Italy).

${ }^{1}$ A. Hubert and R. Schäfer, Magnetic Domains (Springer, Berlin, 1998).

${ }^{2}$ M. Kläui, C. A. F. Vaz, J. A. C. Bland, T. L. Monchesky, J. Unguris, E. Bauer, S. Heun, S. Cherifi, A. Locatelli, and L. J. Heyderman, Phys. Rev. B 68, 134426 (2003).

${ }^{3}$ S. McVietie, G. S. White, J. Scott, P. Warin, and J. N. Chapman, J. Appl. Phys. 90, 5220 (2001).

${ }^{4}$ A. Wachowiak, J. Wiebe, M. Bode, O. Pietzsch, M. Morgenstern, and R. Wiesendanger, Science 298, 577 (2002).

${ }^{5}$ P.-O. Jubert, R. Allenspach, and A. Bischof, Phys. Rev. B 69, 220410 (2004).

${ }^{6}$ P. Bruno, Phys. Rev. Lett. 83, 2425 (1999).

${ }^{7}$ M. Kläui, C. A. F. Vaz, L. Lopez-Diaz, and J. A. C. Bland, J. Phys.: Condens. Matter 15, 985 (2003).

${ }^{8}$ M. Kläui, C. A. F. Vaz, W. Wernsdorfer, E. Bauer, S. Cherifi, S. Heun, A. Locatelli, G. Faini, E. Cambril, L. J. Heyderman, and J. A. C. Bland, Physica B 343, 343 (2004).

${ }^{9}$ C. A. F. Vaz, L. Lopez-Diaz, M. Kläui, T. L. Monchesky, J. Unguris, Z. Cui, and J. A. C. Bland, Phys. Rev. B 67, 140405 (2003).

${ }^{10}$ M. Kläui, C. A. F. Vaz, J. Rothman, J. A. C. Bland, W. Wernsdorfer, G. Faini, and E. Cambril, Phys. Rev. Lett. 90, 097202 (2003).

${ }^{11}$ R. D. McMichael and M. J. Donahue, IEEE Trans. Magn. 33, 4167 (1997).

${ }^{12}$ J. Grollier, P. Boulenc, V. Cros, A. Hamzić, A. Vaurès, and A. Fert, Appl. Phys. Lett. 83, 509 (2003).

${ }^{13}$ M. Kläui, C. A. F. Vaz, J. A. C. Bland, W. Wernsdorfer, G. Faini, E. Cambril, and L. J. Heyderman, Appl. Phys. Lett. 83, 105 (2003).

${ }^{14}$ F. J. Castano, C. A. Ross, C. Frandsen, A. Eilez, D. Gil, H. I. Smith, M. Redjdal, and F. Humphrey, Phys. Rev. B 67, 184425 (2003).

${ }^{15}$ S. P. Li, D. Peyrade, M. Natali, A. Lebib, Y. Chen, U. Ebels, L. D. Buda, and K. Ounadjela, Phys. Rev. Lett. 86, 1102 (2001).

${ }^{16}$ J. Rothman, M. Kläui, L. Lopez-Diaz, C. A. F. Vaz, A. Bleloch, J. A. C. Bland, Z. Cui, and R. Speaks, Phys. Rev. Lett. 86, 1098 (2001).

${ }^{17}$ M. Kläui, C. A. F. Vaz, A. Lapicki, T. Suzuki, Z. Cui, and J. A. C. Bland, Microelectron. Eng. 73, 785 (2004).

${ }^{18}$ L. J. Heyderman, C. David, M. Kläui, C. A. F. Vaz, and J. A. C. Bland, J. Appl. Phys. 93, 10011 (2003).

${ }^{19}$ Y. G. Yoo, M. Kläui, C. A. F. Vaz, J. A. C. Bland, and L. J. Heyderman, Appl. Phys. Lett. 82, 2470 (2003).

${ }^{20}$ A. Locatelli, S. Cherifi, S. Heun, M. Marsi, K. Ono, A. Pavlovska, and E. Bauer, Surf. Rev. Lett. 9, 171 (2002).

${ }^{21}$ The free oOMMF package is available at http://math.nist.gov/oommf

${ }^{22}$ R. P. Cowburn, D. K. Koltsov, A. O. Adeyeye, and M. E. W. D. M. Tricker, Phys. Rev. Lett. 83, 1042 (1999).

${ }^{23}$ M. Kläui, C. A. F. Vaz, J. A. C. Bland, E. H. C. P. Sinnecker, A. P. Guimaraes, W. Wernsdorfer, G. Faini, E. Cambril, L. J. Heyderman, and C. David, Appl. Phys. Lett. 84, 951 (2004). 